\title{
Simultaneous Improvement of Energy Efficiency and Product Quality in PCB Lamination Process
}

\author{
You-Jin Park ${ }^{1,4}$, Rong Pan², Connie M. Borror ${ }^{3}$, Douglas C. Montgomery'2, and Gyu-Bong Lee \\ 1 School of Business Administration, College of Business and Economics, Chung-Ang University, Heukseok-dong, Dongjak-gu, Seoul, South Korea, 156-756 \\ 2 School of Computing, Informatics, Decision Systems Engineering, Ira A. Fulton School of Engineering, Arizona State University, 699 South Mill Avenue, Tempe, AZ, USA, 85281 \\ 3 School of Mathematical and Natural Sciences, New College of Interdisciplinary Arts and Sciences, Arizona State University West, 37100, Phoenix, AZ, USA, 85069 \\ 4 Manufacturing Service Technology Center, Korea Institute of Industrial Technology, 35-3, Hongcheon-ri, Ipjang-myeon, Cheonan-si, Chungcheongnam-do, 330-825 \\ \# Corresponding Author / E-mail: eugenepark@cau.ac.kr, TEL: +82-31-670-3681, FAX: +82-31-675-1384
}

KEYWORDS: Lamination process, PCB manufacturing, Energy consumption, Non-defective probability, Multi-objective optimization problem, Genetic algorithm

Recently, efficient use of energy resources has become a very critical issue in most industries due to various reasons such as the high price of energy resources and environmental problems. The printed circuit board (PCB) industry is known as one of the major manufacturing industries that consumes a moderately large amount of electricity. Of all PCB manufacturing processes, the photolithography process is the most complicated. The photolithography process consists of: 1) a lamination process; 2) an exposure process; and, 3) a development process. Particularly, the lamination process appears to consume the most energy among the entire PCB manufacturing processes. This is due to the use of high temperatures and high pressures in that process which are required to employ photo sensitive dry film resist-coating on the panel. In addition, the PCB panel quality after the lamination process is highly dependent on conditions of three main operating parameters, temperature, pressure, and conveyor belt speed. In this research, we employ designed experiments and model-building techniques to obtain optimal settings of the three main operating parameters which will simultaneously minimize energy consumption while maximizing the probability of creating a non-defective PCB panel during the lamination process.

\section{NOMENCLATURE}

\author{
$x_{1}=$ Temperature \\ $x_{2}=$ Pressure \\ $x_{3}=$ Conveyor belt speed \\ $y_{1}=$ Energy consumption \\ $y_{2}=$ Non-defectiveness (PCB panel quality) \\ $\pi(\mathbf{x})=$ Non-defective probability \\ $\mathbf{x}=$ Vector of operating parameters (temperature, pressure, conveyor \\ belt speed)
}

\section{Introduction}

A major challenge for most manufacturing companies is to improve productivity or product quality without significantly increasing production cost. In recent years, efficient use of energy resources and development of energy usage methods have become very important issues in all aspect of manufacturing. Efficient usage of energy resources is necessary due to high prices of energy resources and serious environmental problems such as global warming. It is known that energy consumed in manufacturing processes accounts for a large proportion of energy used in all sectors of an industry or company. As a result, energy consumption is one of the most significant factors directly influencing overall manufacturing costs across an industry. It is important to improve energy efficiency (or reduce energy consumption) resulting in the reduction of production costs while maintaining required levels of the key production performance measures.

In this research, we consider the lamination process (a sub-process of the photolithography process) since it is one of the most energyguzzling processes in printed circuit board (PCB) manufacturing. Our goal is to find an optimal operating condition which minimizes energy consumption while maximizing the probability of creating a highquality PCB panel during the lamination process (i.e., maximize the non-defective probability). We construct appropriate, but separate 
regression models for modeling energy consumption behavior and modeling the non-defective probability with using data through a novel experimental design technique, and apply two simple heuristic optimization methods to obtain an optimal operating condition. We provide a numerical analysis to validate the optimization methods used, and also compare the performance of two proposed methods through examining the quality of solutions.

\section{Printed Circuit Board Manufacturing Processes}

\subsection{Lamination Process}

Printed circuit boards (PCBs) are used in most electronic products that require moderately high-level technologies and provide the electrical interconnections between microelectronic components such as semiconductor chips and capacitors mounted on the board to create an electronic system. ${ }^{1}$ Because the density of the PCB is increasing with the progress of PCB manufacturing technology, there has been a growing concern about current modeling and analysis techniques used for PCB manufacturing processes. Generally, the PCB manufacturing process consists of several complicated operations such as bonding, drilling, photolithography, metallizing, plating, etching and striping, coating and finishing, and inspection. Fig. 1 displays the general manufacturing process for PCBs.

In the PCB manufacturing process, the photolithography process is considered the most important since this process transfers an image or pattern onto a PCB panel. The photolithography process consists of three sub-processes: 1) lamination process applying photoresist; 2) exposure process exposing to ultraviolet (UV) light; and 3) development process for developing the image. ${ }^{2,3}$ Among these three sub-processes, the lamination process requires moderately high electric energy for heating or cooling, maintaining a certain level of pressure, and controlling the predetermined conveyor movement (speed). In order to reduce the energy consumption of the photolithography process, electricity

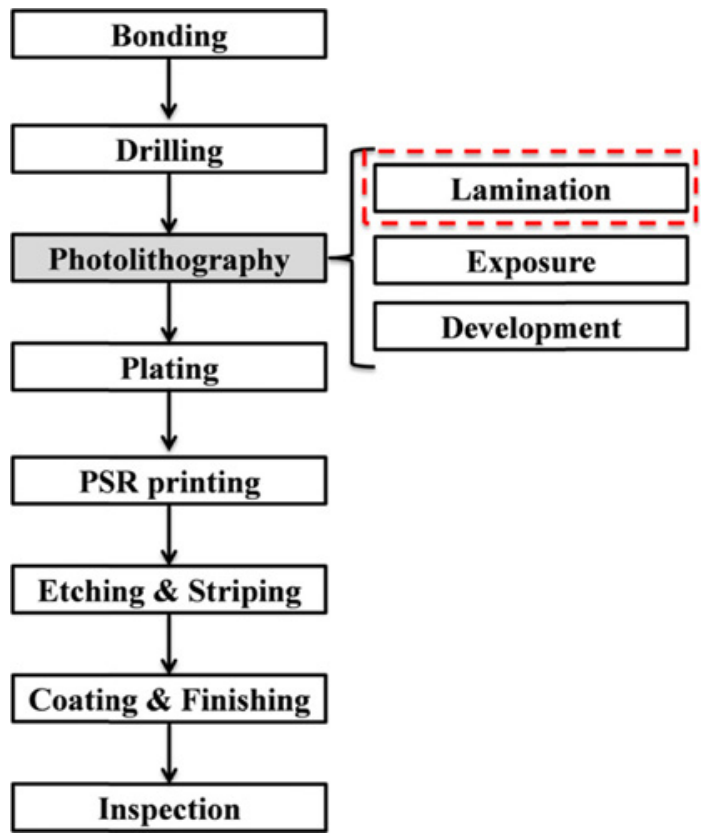

Fig. 1 Printed circuit board manufacturing process consumed during the lamination process should be minimized while maintaining a certain level of product quality or productivity. However, it is often difficult to determine appropriate operating conditions which simultaneously satisfy these two criteria. In the next sections, we present various optimization problems as well as energy saving techniques that can be used in PCB manufacturing processes.

\subsection{Energy Savings in PCB Manufacturing Process Simulation}

Due to the high costs of natural resources and serious environmental issues, endeavors to reduce energy consumption and enhance energy efficiency in various fields of industry are increasing. Like all other industries, energy savings in PCB manufacturing is also becoming an important concern with the rapid growth of $\mathrm{PCB}$ manufacturing technology. ${ }^{4}$ Some energy-saving techniques and the best practices for the PCB industry have been introduced. However, there has been little research on energy savings and energy reduction methodologies for PCB manufacturing processes. An extensive literature review on energy consumption reduction strategies, energy policies, and the stateof-the-art for energy-saving methods that are currently being pursued in several major countries has been conducted. ${ }^{5}$ For sustainable manufacturing, Lee et al. (2012) developed a simulation-based analysis system prototype to assess the impact of manufacturing systems on the environment. The system prototype has been applied to a case study with various scenarios for "what-if" analyses. ${ }^{6}$ Goosey and Kellner (2010) presented details of key best practices that can lead PCB manufacturing companies to optimize energy consumption, conserve materials, and reduce waste and costs. ${ }^{7}$

A difference in energy consumption patterns of a conventional CNC machine and a micro-milling machine has also been analyzed. ${ }^{8}$ Luo et al. (2005) conducted a theoretical and experimental study on finding intrinsic relationships between tool flank wear and operational conditions in metal cutting processes. ${ }^{9}$ In order to optimize the energy footprint for a machined product, Rajemi et al. (2010) provided a new model and methodology using key critical parameters which affect energy usage and the environmental footprint. ${ }^{10}$ On the machining technology level, general issues, a new method, and a case study for achieving production sustainability has been completed. ${ }^{11}$ In the next section, a discussion on current research on optimization issues in PCB manufacturing processes will be provided.

In order to improve the energy efficiency in PCB manufacturing processes, an energy resource management (ERM) system has been proposed which allows energy consumption and $\mathrm{CO}_{2}$ emissions to be managed and analyzed efficiently. The ERM system is designed to measure and collect the energy usage data in real time through an electric power meter known as a machine-to-machine (M2M) device. The system has been applied to inner layer scrubbing processes and drilling processes for PCB fabrication and the reflow soldering process for surface mounting of chips or devices on PCB. ${ }^{12}$ Park and Dang (2012) applied a heuristic approach and experimental design technique to an in-line induction heating process in automotive crankshaft manufacturing in order to find an optimal operating condition that can improve energy efficiency. They considered voltage and frequency as the key operating parameters and devised a proper heating strategy. In addition, thermal insulation has been proposed as a tool for reducing heat losses in this process. ${ }^{13}$ 


\subsection{Optimization Problems in PCB Manufacturing Processes}

There are many complicated problems which could not be solved easily in various electronics manufacturing systems, including PCB and semiconductor device manufacturing, electronics assembly, etc. In order to improve productivity and product quality, a series of problems encountered within the entire manufacturing process should be formulated and solved simultaneously. It is necessary to develop and utilize effective analysis and control approaches such as response surface methodology which involves various useful optimization techniques as well as experimental design techniques.

The problems of sequencing, assignment, and balancing which arises under various machine architectures in the automated PCBs production system were modeled based on a decomposition leading to travelling salesman, rural postman, assignment, and quadratic assignment or line balancing problems. ${ }^{14}$ An integrated approach including a mathematical model has been developed for solving the problem of assigning component types to feeder locations and sequencing component placements on the PCB in order to minimize the total head movement when component types are allowed to be placed in multiple feeders. ${ }^{15}$ Doniavi et al. (2000) developed a systematic generic model integrated with a computer aided manufacturing (CAM) technique which can help PCB manufacturers obtain a process map for PCBs and find optimal settings for photolithography equipment, and consequently increase the process yield. ${ }^{16}$

Ancău (2008) attempted to improve PCB manufacturing process productivity through the reduction of tool path length and processing time in a drilling process. In order to reduce the tool path length, Ancãu (2008) devised an original hybrid heuristic algorithm based on the structure of a general optimization procedure and developed a practical mathematical model for calculation of PCB processing time. This study showed the influence of stacked PCBs on process productivity and provided a guide for finding the optimal length of the tool path as well as the optimal number of stacked PCB's for efficient drilling. ${ }^{17}$ Ancãu (2009) also considered and optimized two key performance measures to enhance the productivity of the PCB drilling operation. The first performance measure is the cutting-tool path length which affects the total processing time, and the second metric is the number of stacked PCBs which affects processing efficiency. A heuristic hybrid algorithm based on the travelling salesman problem (TSP) was used to minimize the cutting-tool path length between the holes of a PCB and then find the optimal number of stacked PCBs that can be properly processed. ${ }^{18}$ Also, ant colony optimization (ACO) algorithm was proposed to solve the $\mathrm{PCB}$ drilling problem. ${ }^{19} \mathrm{~A}$ systematic approach was developed to reduce the standard deviation and adjust the mean specification of flexible printed circuit boards (FPCBs) widths which is used for lighting in automotive vehicles. The quality performances or responses of lighting vehicles depend upon the circuit width of an FPCB and the etched rate of an acid solution. The approach includes three sequential steps involving experimental design techniques, steepest descent methods, and interchangeable linear constrained response surface optimization models (IC-LCRSOM). ${ }^{20}$

Compared to other processes of PCB manufacturing, there have been many approaches developed for optimizing PCB assembly since product variety and complexity has increased. As a result, operational efficiency in PCB manufacturing industry has become a very critical issue. A PCB assembly task requires that a set of components be picked from their respective pickup locations and then be placed at their respective placement locations on the board being assembled. ${ }^{21}$ Therefore, the assembly of PCBs is often a complex task which places hundreds of electronic components of different shapes and sizes at specific locations on the board. The efficiency of the component placement process in PCB assembly depends on in part, how to determine the appropriate component placement sequence and to assign component types to feeders of the placement machine. Efficient assembly of PCBs through automation and optimization of the process is essential for reducing production cost and increasing productivity. ${ }^{22}$

In order to reduce overall assembly time in an automatic PCB assembly machine with a robot arm, an integer programming (IP) approach has been developed. This approach is designed to find optimal values of both the pickup locations of the components and the sequence in which the pickup and placement of components is performed. Through this approach, it has been possible to obtain near optimal solutions which are computationally tractable using the techniques of (i) minimum weight matching for determining an optimal assignment of pickup locations and (ii) traveling salesman problem (TSP) for determining an optimum sequence of pickups and placements. ${ }^{21}$

Altinkemer et al. (2000) considered the problem of allocating the components to feeders and determining the placement sequence of the components on the PCBs in a situation where a rotary head machine with surface mount technology is used. They considered all subproblems simultaneously and integrated the sub-problems into a single problem, and then applied an $\varepsilon$-approximation algorithm which is often used in conventional vehicle routing problems. ${ }^{23}$

A PCB assembly planning method has been proposed to improve the productivity of a gantry-type machine with multiple assembly heads. Park and Kim (2007) formulated the assembly planning optimization problem as an integer programming problem. They developed a heuristic algorithm involving dynamic programming techniques and applied to both the feeder arrangement optimization and placement sequence optimization problems in order to reduce the overall assembly time. ${ }^{24}$

Ho et al. (2008) developed two hybrid genetic algorithms to minimize the total placement time spent by the revolver head for assembling all components on the PCB. Using these algorithms, they solved three component placement problems simultaneously for the collect-and-place (CAP) machine with one revolver head used in a PCB assembly process. That is, a component grouping problem, a component sequencing problem, and a feeder arrangement problem. They proved that better final solutions as well as better initial solutions could be generated through the use of the algorithms hybridized with several heuristic approaches. For example, hybridization of the Clarke and Wright saving method, the nearest neighbor heuristic method, and neighborhood frequency heuristic method. ${ }^{25}$ In next section, we will present the problem considered in this research and statistical models for both energy consumption and nondefective probability of PCB panels processed in the lamination stage.

\section{Optimization Methods}

\subsection{Multi-objective Optimization Problem}

The problem considered in this research is a case of multi-objective decision making since it involves two objective functions to be optimized simultaneously. Many innovative theories and techniques for 
dealing with diverse multi-objective optimization problems have been discussed and applied to real problems in engineering, science, and economics; where a certain optimal decision needs to be made where trade-offs among more than two conflicting criteria exist.

For a nontrivial multi-objective optimization problem, there may not be a single solution that simultaneously optimizes each objective. In that case, the objective functions are said to be conflicting, with possibly an infinite number of Pareto optimal solutions. A solution is called non-dominated, Pareto optimal, Pareto efficient or non-inferior, if none of the objective functions can be improved in value without degrading some of the other objective values. Generally, the main goal of a multi-objective optimization analysis is to find a representative set of Pareto optimal solutions, quantify the trade-offs in satisfying the different objectives, and then determine a single solution that satisfies the subjective preferences of a human decision maker.

The most widely used methods for solving multi-objective optimization problems are the $\varepsilon$-constraint method and weighted sum method which can provide a representative subset of the Pareto set and in most cases is adequate. The $\varepsilon$-constraint method proposed by Haimes et al. (1971) is used to generate Pareto optimal solutions. ${ }^{26}$ This approach makes use of a single-objective optimizer that handles constraints, to generate one point of the Pareto front at a time. For transforming the multi-objective problem into several single-objective problems with constraints, it uses the following procedure for a problem in which all the objective functions should be minimized:

$$
\begin{array}{ll}
\min & f_{1}(\mathbf{x}) \\
\text { s.t. } & f_{1}(\mathbf{x}) \leq \varepsilon_{j}, j=1,2, \ldots, m, j \neq l \\
& \mathbf{x} \in S
\end{array}
$$

where $l \in\{1,2, \ldots, m\}$ and $S$ is the feasible region, which can be defined by any equality and/or inequality constraint. The vector of upper bounds, $\varepsilon \in\left\{\varepsilon_{1}, \varepsilon_{2}, \ldots, \varepsilon_{m}\right\}$, defines the maximum value for each objective. The vector of upper bounds should be changed along the Pareto front for each objective to obtain a subset of the Pareto optimal set, and a new optimization procedure for the new vector of upper bounds should be constructed. In other words, this method optimizes one objective function and simultaneously maintains the maximum or minimum acceptable levels for the other objective functions. As an advanced version of the conventional $\varepsilon$-constraint method, the augmented $\varepsilon$-constraint (AUGMECON) method has been designed to avoid the production of poor Pareto optimal solutions and accelerate the entire process by avoiding redundant iterations. ${ }^{27}$

The weighted sum method is the simplest approach and the most widely used classical method. It scalarizes the set of objectives into a single objective by multiplying each objective by a weight. This approach works well when the objectives are well behaved and tradeoffs between the objectives allow the weights to be easily determined ahead of time. ${ }^{28}$ Additionally, an adaptive weighted sum method for multi-objective optimization problems has been developed, which determines uniformly-spaced Pareto optimal solutions, finds solutions on non-convex regions, and neglects non-Pareto optimal solutions. ${ }^{29}$ The goal programming approach has been proposed for minimizing deviations from individual goals and then an evolutionary optimization algorithm has been suggested to find multiple Pareto-optimal solutions of the resulting multi-objective optimization problem. ${ }^{30}$

For simultaneously optimizing two objectives, the dual-response surface methodology is known to be a powerful tool and has been successfully used for optimization in various cases. ${ }^{31}$ Vining and Myers (1990) shows how the dual response approach of optimizing a primary response function while satisfying conditions on a secondary response function can be used to achieve the goals. ${ }^{32}$ The desirability function optimization approach is also one of the most widely used methods in many industries for the optimization of multiple responses. For each response, $y_{i}$, a desirability function $d_{i}\left(y_{i}\right)$ assigns a number between 0 and 1 to each possible value of $y_{i}$, with $d_{i}\left(y_{i}\right)=0$ representing a completely undesirable value of $y_{i}$ and $d_{i}\left(y_{i}\right)=1$ representing a completely desirable or ideal response value. The individual desirability is then combined using the geometric mean, which gives the overall desirability $D$ :

$$
D=\sqrt[k]{d_{1}\left(y_{1}\right) \times d_{2}\left(y_{2}\right) \times \ldots \times d_{k}\left(y_{k}\right)}
$$

with $k$ denoting the number of responses. Notice that if any response $y_{i}$ is completely undesirable, then the overall desirability is zero. In practice, fitted response values $\hat{y}_{i}$ are used in place of the $y_{i}{ }^{33}$

\subsection{Heuristic Approaches}

It is known that the $\varepsilon$-constraint method has several advantages over the weighted sum method. However, when the $\varepsilon$-constraint method is applied to the multi-objective problem, this method has three critical drawbacks: (1) it does not generate a set of non-dominated solutions in a single run; (2) appropriate upper bounds for the $\varepsilon_{j}$ values must be provided; and, (3) it requires additional constraints to be satisfied. Moreover, the method is not particularly efficient as the number of the objective functions increases. The well-known drawbacks of the weighted sum method are: (1) its general design is not guaranteed to be Pareto optimal, (2) a satisfactory and a priori selection of weights does not necessarily guarantee that the final solution will be acceptable even though there are many methods for determining weights, (3) it is impossible to obtain points on non-convex portions of the Pareto optimal set in the criterion space, and (4) varying the weights consistently and continuously may not necessarily result in an even distribution of Pareto optimal points and an accurate, complete representation of the Pareto optimal set. In addition, the fundamental weakness of goal programming approach is to produce solutions that are not Pareto efficient.

Compared to these methods, heuristic approaches to solve multiobjective optimization problems with two or more objective functions have become very popular. Many useful heuristic methods have evolved to facilitate solving optimization problems which were previously difficult or impossible to solve. These methods include simulated annealing, tabu search, particle swarm, evolutionary algorithms, etc. In mechanics, a physical process called annealing is performed in order to relax the system to a state with minimum free energy. Based on the annealing process in the mechanics, the simulated annealing (SA) algorithm has been introduced for solving complicated combinatorial optimization problems. Usually, the SA algorithm helps avoid staying at a local optimum and to rapidly reach the vicinity of an optimal solution. Tabu search (TS) algorithm is a gradient-descent 
search with memory. The memory preserves a number of previously visited states along with a number of states that might be considered unwanted. This information is stored in a tabu list. Using the length of tabu list, aspiration, and diversification, it searches the better solutions of optimization problems. Evolutionary algorithms are methods based on ideas of biological evolution for searching the solution of an optimization problem. They utilize the principle of survival in order to produce gradual approximations to the optimum. A new set of approximations is created through the process of selecting individuals according to their objective function, which is called fitness for evolutionary algorithms, and breeding them together using operators inspired from genetic processes.

The most successful evolutionary algorithms are genetic algorithms (GAs) that mimic the process of natural evolution. ${ }^{34}$ Genetic algorithms developed by Holland (1992) seek solutions of the problems through recombination operators such as cloning (inheritance), mutation, crossover, etc. ${ }^{34,35}$ The recombination operators are designed to generate useful solutions to optimization and search problems. In a genetic algorithm, a population of candidate solutions to an optimization problem is evolved toward better solutions. Each candidate solution has a set of properties which can be represented in an encodings. In each generation, the fitness of every individual solution in the population is evaluated. The more fit individuals are selected from the current population, and each individual's genome is modified to form a new generation. Commonly, the algorithm terminates when either a maximum number of generations has been produced, or a satisfactory fitness level has been reached for the population. ${ }^{36}$ Due to the computational efficiency, the prevalent area for applying genetic algorithms are optimization problems requiring large scale high performance computing resources. ${ }^{37}$

\section{Model Development}

Through the lamination process, photo-sensitive dry film resists are coated on the top and bottom surfaces of PCB panels. In the lamination process, appropriate operating conditions of three key parameters (factors) should be determined to manufacture qualified products. The three key parameters are temperature, pressure, and conveyor belt speed. There are also two important responses of interest or measures of process quality influenced by the key operation parameters in a PCB lamination process. These responses are energy consumption (electricity) $\left(y_{1}\right)$ and panel quality $\left(y_{2}\right)$ (panel quality is a binary response, defective or non-defective). The minimum and maximum limits of three operations parameters considered in PCB lamination process are shown in Table 1.

It is important to identify optimal settings of these operation parameters which can simultaneously reduce energy consumption and improve panel quality. For a binary response such as panel quality, the probability of an outcome (such as a panel being non-defective) can be used as the response to build a model representing panel quality.

Since we have two very different responses, one is continuous while the other categorical, two different modeling approaches are necessary. For energy consumption, $y_{1}$, we wanted to investigate a broad relationship between the response and the three input parameters,
Table 1 Minimum and maximum limits of temperature, pressure and conveyor belt speed (CBS)

\begin{tabular}{cccccc}
\hline \multicolumn{2}{c}{ Temperature $\left({ }^{\circ} \mathrm{C}\right)$} & \multicolumn{2}{c}{ Pressure $(\mathrm{bar})$} & \multicolumn{2}{c}{ CBS $(\mathrm{m} / \mathrm{min})$} \\
\hline Min & Max & Min & Max & Min & Max \\
\hline 100 & 120 & 3.0 & 6.0 & 1.0 & 5.0 \\
\hline
\end{tabular}

temperature $\left(x_{1}\right)$, pressure $\left(x_{2}\right)$, and conveyor belt speed $\left(x_{3}\right)$. Specifically, for investigating curvature in the true response surface, a full second-order model in the three parameters is of interest to be fit:

$$
\begin{aligned}
& y=\beta_{0}+\beta_{1} x_{1}+\beta_{2} x_{2}+\beta_{3} x_{3}+\beta_{12} x_{1} x_{2}+\beta_{13} x_{1} x_{3} \\
&+ \beta_{23} x_{2} x_{3}+\beta_{11} x_{1}^{2}+\beta_{22} x_{2}^{2}+\beta_{33} x_{3}^{2}+\varepsilon
\end{aligned}
$$

where $\beta_{0}, \beta_{1}, \beta_{2}, \beta_{3}, \beta_{12}, \beta_{13}, \beta_{23}, \beta_{11}, \beta_{22}$ and $\beta_{33}$ denote the intercept, regression coefficients for linear effects $x_{1}, x_{2}$, and $x_{3}$, regression coefficients for interaction effects $x_{12}, x_{13}$, and $x_{23}$, and regression coefficients for pure quadratic effects, $x_{1}^{2}, x_{2}^{2}$ and $x_{3}^{2}$, respectively. In addition, $\varepsilon$ represents error where $\varepsilon \sim N\left(0, \sigma^{2}\right){ }^{38}$

For panel quality, it is necessary to employ a logistic regression model to explain the effect of three operation parameters on quality of PCB panels (degree of defectiveness) of lamination process. A logistic regression is most appropriate since the response variable is categorical resulting in an outcome of either 0 or 1 (i.e., bad or good). In general statistical analysis, logistic regression analysis is used for predicting the probability of an outcome when several explanatory variables are involved. ${ }^{39}$ A general logistic regression model with three independent variables is represented as follows:

$$
\pi\left(x_{1}, x_{2}, x_{3}\right)=\frac{\exp \left(\gamma_{0}+\gamma_{1} x_{1}+\gamma_{2} x_{2}+\gamma_{3} x_{3}\right)}{1+\exp \left(\gamma_{0}+\gamma_{1} x_{1}+\gamma_{2} x_{2}+\gamma_{3} x_{3}\right)}
$$

where $\pi(\bullet)$ represents the probability a single trial is good, and $\gamma_{0}, \gamma_{1}, \gamma_{2}$ and $\gamma_{3}$ denote the intercept and regression coefficients for linear effects $x_{1}, x_{2}$, and $x_{3}$, respectively. Using vector notation, we let $\mathbf{x}=\left[x_{1}, x_{2}, x_{3}\right]$, and rewrite the above equation as

$$
y_{2}=\ln \left(\frac{\pi(\mathbf{x})}{1-\pi(\mathbf{x})}\right)=\gamma_{0}+\gamma_{1} x_{1}+\gamma_{2} x_{2}+\gamma_{3} x_{3}
$$

Various combinations of levels for the three explanatory variables are needed in order to support the full second-order model for $y_{1}$ and the logistic regression model for $y_{2}$. A single designed experiment can be constructed for this purpose. The design used in this study is a $3^{3}$ factorial design with 3 center-points which will allow for estimation of all terms in the second-order model as well as the logistic regression model. ${ }^{39}$ The design and the resulting experimental data for $y_{1}$ and $y_{2}$ are displayed in Table 2 .

In Table 2, $x_{1}, x_{2}, x_{3}, y_{1}$, and $y_{2}$ denote temperature, pressure, conveyor belt speed, energy consumption, and panel quality, respectively.

It is often of interest to graphically examine results, in particular when there are two or more responses. Fig 2 displays the response, energy consumption against run order. In addition, the panel quality corresponding to each run is denoted by trapezoid-shape $\left(y_{2}=0\right)$ or square-shape $\left(y_{2}=1\right)$. Recall $y_{2}=1$ indicates a good quality panel. From Fig. 2 we see that there is almost no significant correlation between good and bad panels and energy consumption; defective 
Table 2 Experimental data of energy consumption and PCB panel quality

\begin{tabular}{|c|c|c|c|c|c|}
\hline No. & $x_{1}$ & $x_{2}$ & $x_{3}$ & $y_{1}$ & $y_{2}$ \\
\hline 1 & 100 & 3 & 1 & 0.706046088 & 0 \\
\hline 2 & 110 & 3 & 1 & 0.681832846 & 1 \\
\hline 3 & 120 & 3 & 1 & 1.176922985 & 1 \\
\hline 4 & 100 & 4.5 & 1 & 0.846171391 & 0 \\
\hline 5 & 110 & 4.5 & 1 & 0.917131781 & 1 \\
\hline 6 & 120 & 4.5 & 1 & 0.923457828 & 1 \\
\hline 7 & 100 & 6 & 1 & 0.894828623 & 0 \\
\hline 8 & 110 & 6 & 1 & 0.717099480 & 1 \\
\hline 9 & 120 & 6 & 1 & 1.150222049 & 0 \\
\hline 10 & 100 & 3 & 3 & 0.734446081 & 1 \\
\hline 11 & 110 & 3 & 3 & 0.838744360 & 1 \\
\hline 12 & 120 & 3 & 3 & 1.181558457 & 1 \\
\hline 13 & 100 & 4.5 & 3 & 0.895516788 & 1 \\
\hline 14 & 110 & 4.5 & 3 & 0.995199901 & 1 \\
\hline 15 & 120 & 4.5 & 3 & 0.986872605 & 1 \\
\hline 16 & 100 & 6 & 3 & 0.689764396 & 0 \\
\hline 17 & 110 & 6 & 3 & 0.885586201 & 1 \\
\hline 18 & 120 & 6 & 3 & 1.146313010 & 0 \\
\hline 19 & 100 & 3 & 5 & 0.509364831 & 0 \\
\hline 20 & 110 & 3 & 5 & 1.054686682 & 1 \\
\hline 21 & 120 & 3 & 5 & 1.476025077 & 1 \\
\hline 22 & 100 & 4.5 & 5 & 0.679702677 & 0 \\
\hline 23 & 110 & 4.5 & 5 & 1.155098004 & 1 \\
\hline 24 & 120 & 4.5 & 5 & 1.362611735 & 1 \\
\hline 25 & 100 & 6 & 5 & 1.118997682 & 0 \\
\hline 26 & 110 & 6 & 5 & 1.498364052 & 0 \\
\hline 27 & 120 & 6 & 5 & 1.959743959 & 0 \\
\hline 28 & 110 & 4.5 & 3 & 0.940385727 & 1 \\
\hline 29 & 110 & 4.5 & 3 & 0.985267751 & 1 \\
\hline 30 & 110 & 4.5 & 3 & 0.923811939 & 1 \\
\hline
\end{tabular}

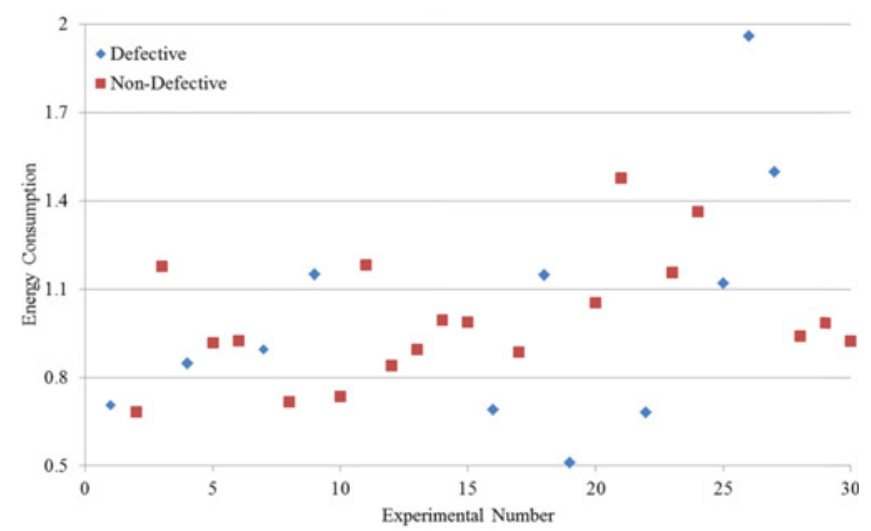

Fig. 2 Experimental results of energy consumption and panel quality by run order

panels are associated with both the highest level of energy consumption (experiment \#27) but also one of the lowest energy consumption levels (experiment \#19). In addition, we can see that non-defective panels (denoted in red) are more frequent and appear often with lower levels of energy consumption.

After a brief graphical description of the responses, an analysis of variance is carried out for energy consumption with results displayed in Table 3. They null hypothesis begin tested is none of the regression
Table 3 ANOVA of the regression model for energy consumption

\begin{tabular}{cccccc}
\hline Source & df & SS & MS & $F$-value & $p$-value \\
\hline Regression & 9 & 2.12436 & 0.23604 & 10.79 & 0.0000 \\
\hline Error & 20 & 0.43759 & 0.02188 & & \\
\hline Total & 29 & 2.56196 & & & \\
\hline
\end{tabular}

Table 4 Estimates of regression coefficients and test statistics

\begin{tabular}{ccccc}
\hline Predictor & $\beta$ & $\mathrm{SE}(\beta)$ & $t$-value & $p$-value \\
\hline Intercept & 0.89917 & 0.05649 & 15.92 & 0.000 \\
\hline$x_{1}$ & 0.23827 & 0.03486 & 6.83 & 0.000 \\
\hline$x_{2}$ & 0.09452 & 0.03486 & 2.71 & 0.013 \\
\hline$x_{3}$ & 0.15560 & 0.03486 & 4.46 & 0.000 \\
\hline$x_{12}$ & -0.02766 & 0.04270 & -0.65 & 0.524 \\
\hline$x_{13}$ & 0.14056 & 0.04270 & 3.29 & 0.004 \\
\hline$x_{23}$ & 0.11164 & 0.04270 & 2.61 & 0.017 \\
\hline$x_{11}$ & 0.03595 & 0.05649 & 0.64 & 0.532 \\
\hline$x_{22}$ & 0.03295 & 0.05649 & 0.58 & 0.566 \\
\hline$x_{33}$ & 0.10091 & 0.05649 & 1.79 & 0.089 \\
\hline & & & &
\end{tabular}

Table 5 Analysis of maximum likelihood estimates

\begin{tabular}{ccccc}
\hline Predictor & $\mathrm{g}$ & $\mathrm{SE}(\gamma)$ & $\mathrm{Z}$ & $p$-value \\
\hline Intercept & 0.856062 & 0.505776 & 1.69 & 0.091 \\
\hline$x_{1}$ & 1.487830 & 0.732166 & 2.03 & 0.042 \\
\hline$x_{2}$ & -1.788810 & 0.765423 & -2.34 & 0.019 \\
\hline$x_{3}$ & -0.390421 & 0.633978 & -0.62 & 0.538 \\
\hline
\end{tabular}

Table 6 Goodness-of-fit test results

\begin{tabular}{cccc}
\hline Methods & Chi-Square & df & $p$-value \\
\hline Pearson & 23.6879 & 23 & 0.421 \\
\hline Deviance & 27.2907 & 23 & 0.244 \\
\hline Hosmer-Lemeshow & 4.0189 & 8 & 0.855 \\
\hline
\end{tabular}

coefficients for the full second-order model are statistically different from 0 (i.e., none of the model terms are statistically significant in explaining the response, $y_{2}$ ). Since the $p$-value for the regression model is small ( $p$ value approximately 0 ), we can reject the null hypothesis and conclude that at least one of the model terms is statistically significant. ${ }^{39}$

Unfortunately the analysis of variance does not indicate which term(s) is/are significant. For that information, we examine $t$-test results for individual terms which are displayed in Table 4. Our analysis indicates the factors temperature, pressure, and conveyor belt speed are statistically significant as well as the interaction between temperature and conveyor belt speed $\left(x_{1} x_{3}\right)$ and the interaction between pressure and conveyor belt speed $\left(x_{2} x_{3}\right)$. The final fitted model for energy consumption is then:

$$
\begin{gathered}
\hat{y}_{1}=1.00106+0.23827 x_{1}+0.09452 x_{2}+0.15560 x_{2} \\
+0.14056 x_{1} x_{3}+0.11164 x_{2} x_{3}
\end{gathered}
$$

For the PCB panel quality in the lamination process, as shown in Table 5 , the estimated coefficients for all factors except conveyor belt speed $\left(x_{3}\right)$ have $p$-values less than 0.05 , indicating there is sufficient evidence that the coefficients for temperature $\left(x_{1}\right)$ and pressure $\left(x_{2}\right)$ are not zero. The estimated logistic regression model for the panel quality is then

$$
\hat{y}_{2}=\ln \left(\frac{\pi(\mathbf{x})}{1-\pi(\mathbf{x})}\right)=0.856+1.488 x_{1}-1.789 x_{2}-0.390 x_{3}
$$

where a vector of three operation parameters is $\mathbf{x}=\left[x_{1}, x_{2}, x_{3}\right]$. 
Table 710 best solutions, energy consumptions, non-defective probabilities, and desirability values from genetic algorithm

\begin{tabular}{ccccccccc}
\hline & $x_{1}$ & $x_{2}$ & $x_{3}$ & EC & D_EC & NDP & D_NDP & OD \\
\hline 1 & 114.0648268 & 3.000302401 & 1.515507653 & 0.932179445 & 0.689178012 & 0.971779410 & 0.938270790 & 0.804136554 \\
\hline 2 & 114.0648268 & 3.000302401 & 1.495594382 & 0.932674162 & 0.689010390 & 0.971885820 & 0.938499857 & 0.804136899 \\
\hline 3 & 114.0648268 & 3.000302401 & 1.495822856 & 0.932668372 & 0.689012352 & 0.971884602 & 0.938497233 & 0.804136920 \\
\hline 4 & 114.0648268 & 3.000302401 & 1.502516604 & 0.932499920 & 0.689069425 & 0.971848875 & 0.938420321 & 0.804137271 \\
\hline 5 & 114.0648268 & 3.000302401 & 1.503235197 & 0.932481971 & 0.689075507 & 0.971845037 & 0.938412059 & 0.804137280 \\
\hline 6 & 114.0648268 & 3.000011814 & 1.503235197 & 0.932494789 & 0.689071164 & 0.971854517 & 0.938432468 & 0.804143490 \\
\hline 7 & 114.0648268 & 3.000011814 & 1.503506441 & 0.932488017 & 0.689073458 & 0.971853069 & 0.938429350 & 0.804143493 \\
\hline 8 & 114.0648268 & 3.000011814 & 1.503883189 & 0.932478618 & 0.689076643 & 0.971851057 & 0.938425019 & 0.804143495 \\
\hline 9 & 114.0648268 & 3.000011814 & 1.504673662 & 0.932458921 & 0.689083317 & 0.971846835 & 0.938415931 & 0.804143496 \\
\hline 10 & 114.0627588 & 3.000011814 & 1.504673662 & 0.932419618 & 0.689096633 & 0.971838416 & 0.938397806 & 0.804143500 \\
\hline & & & & & & & &
\end{tabular}

Table 810 best solutions, energy consumptions, non-defective probabilities, and desirability values from random search method

\begin{tabular}{ccccccccc}
\hline & $x_{1}$ & $x_{2}$ & $x_{3}$ & EC & D_EC & NDP & D_NDP & OD \\
\hline 1 & 114.4085153 & 3.002076773 & 1.425252336 & 0.940880119 & 0.686232995 & 0.973550560 & 0.942087137 & 0.804046813 \\
\hline 2 & 113.9475405 & 3.000622207 & 1.309555630 & 0.936182929 & 0.687822124 & 0.972386522 & 0.939578079 & 0.803904590 \\
\hline 3 & 114.7078555 & 3.010847989 & 1.356375074 & 0.947857924 & 0.683875694 & 0.974747100 & 0.944669739 & 0.803764066 \\
\hline 4 & 114.1340677 & 3.000132480 & 1.202536372 & 0.943448703 & 0.685364782 & 0.973678031 & 0.942362102 & 0.803655272 \\
\hline 5 & 113.4823246 & 3.016772956 & 1.456789107 & 0.922209483 & 0.692560412 & 0.969058638 & 0.932423353 & 0.803591626 \\
\hline 6 & 113.6638957 & 3.014943819 & 1.677616160 & 0.920310110 & 0.693205731 & 0.968638775 & 0.931522619 & 0.803577512 \\
\hline 7 & 114.9214785 & 3.019646585 & 1.442927603 & 0.949330846 & 0.683378617 & 0.974855054 & 0.944902921 & 0.803571062 \\
\hline 8 & 114.9170423 & 3.005226616 & 1.225265124 & 0.955878708 & 0.681171055 & 0.976262102 & 0.947944779 & 0.803562409 \\
\hline 9 & 114.1177759 & 3.025002250 & 1.337057768 & 0.937035822 & 0.687533441 & 0.972140726 & 0.939048698 & 0.803509416 \\
\hline 10 & 113.7593279 & 3.026814078 & 1.378655315 & 0.929213897 & 0.690183236 & 0.970365188 & 0.935229087 & 0.803417350 \\
\hline
\end{tabular}

$x_{1}$ : Temperature, $x_{2}$ : Pressure, $x_{3}$ : Conveyor Belt Speed, EC: Energy Consumption, D_EC: Desirability of Energy Consumption, NDP: Non-Defective Probability, D_NDP: Desirability of Non-Defective Probability, OD: Overall Desirability

Consequently, the linear probability that a manufactured panel is nondefective can be represented as follows:

Test statistics (Pearson, Deviance, Hosmer-Lemeshow Statistics) and corresponding $p$-values for model adequacy of the fitted logistic regression model for PCB panel quality are displayed in Table 6. Based on these results, there is no deficiency in fitness since all $p$-values are greater than the significance level of $\alpha=0.05$.

In Section 4, we will provide an overview of optimization methods applied to many fields, while results of our optimization approach will be presented in Section 5. Conclusions are then given in Section 6 .

\section{Computational Results and Analysis}

In this research, we consider the lamination process in PCB manufacturing and attempt to find optimal operating conditions which minimize total energy consumption and maximize non-defective probability of PCB panel simultaneously. In order to obtain an optimal solution, we developed and applied the genetic algorithm (GA) and random search (RS) method, and then compare the performances of these two approaches using desirability function optimization.

In a GA, the number of offspring at each iteration and the total number of iterations are set to 1,000 and 100 , respectively, and the number of solutions generated for random search method is set to the same offspring number, that is, 100,000 . The proportions of cloning, mutation, and crossover operations used in the GA are $0.2,0.6$, and 0.2 , respectively. All these operation parameters in this algorithm are selected from some experimental tests. A solution in this research consists of three chromosomes (temperature, pressure, and conveyor belt speed). We designed the GA to generate one new solution by changing one chromosome randomly in mutation operation and to generate two new solutions by recombining the solutions in crossover operation. The target values of both energy consumption and nondefective probability are set to 0.1 and 1 , respectively. For calculation of the desirability function values, the required upper bound of energy consumption and the lower bound of non-defective probability are set to 5 and 0.1 , respectively. Also, the same weights for each desirability function $\left(w_{1}=w_{2}=2\right)$ are assigned.

Tables 7 and 8 show the 10 best solutions (conditions of three operating parameters) out of 100,000 distinctive solutions. Results include the energy consumption and non-defective probabilities, the desirabilities for the corresponding objective function values, and overall desirabilities obtained from the genetic algorithm and random search method, and Fig. 3 depicts the overall desirability function values of the 10 best solutions obtained from the two approaches.

As shown in Fig. 3, the overall desirability values from the genetic algorithm always outperform those from the random search method. Figs. 4 and 5 display the energy consumption and non-defective probabilities for the 10 best solutions from the GA and RS methods, respectively. From these two figures. We can see that the GA provides operating conditions which ensure uniformity in both energy consumption and non-defective probabilities compared to the RS method. However, it is necessary to develop a method to generate the good initial solutions for further improvement since the performance of genetic algorithms (GA) is affected by initial population strategies. That is, in order to be protected from being stuck in a local optimum, to get close to the global optimum, and to reduce the number of searches within the solution space, we can use a penalty function 


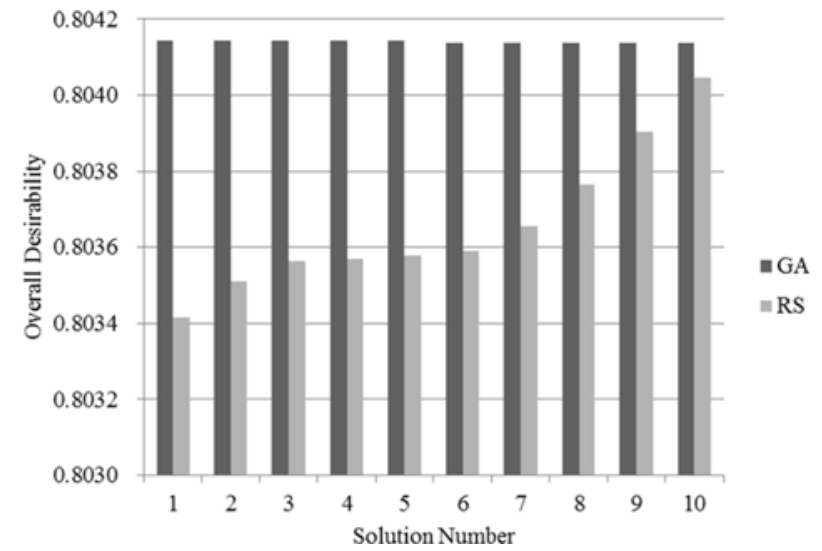

Fig. 3 Overall desirability values for the 10 best solutions from the genetic algorithm (GA) and random search (RS) method

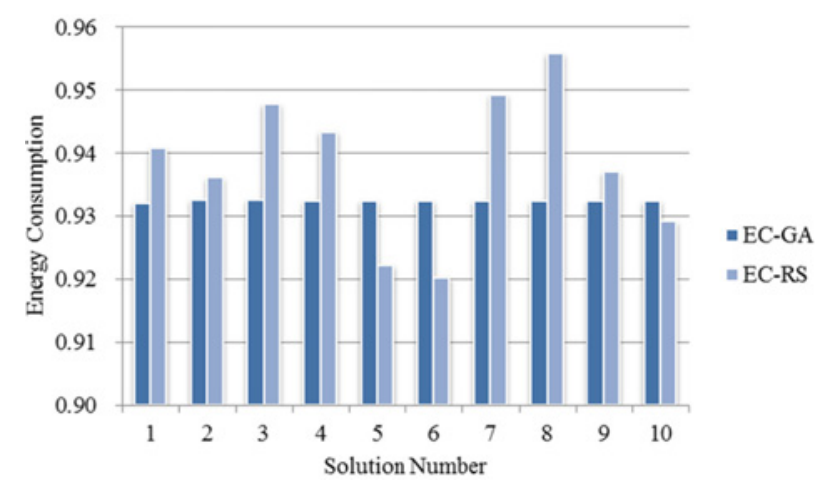

Fig. 4 Energy consumptions for the 10 best solutions from the genetic algorithm (GA) and random search (RS) method

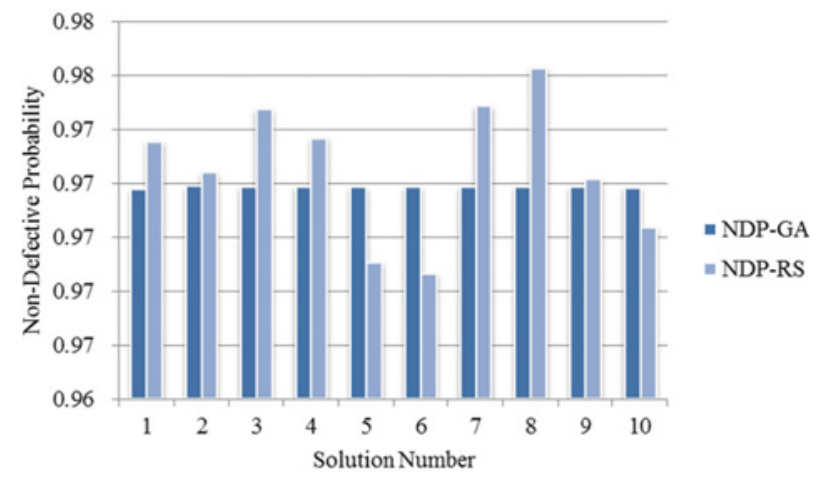

Fig. 5 Non-defective probabilities for the 10 best solutions from the genetic algorithm (GA) and random search (RS) method

method, which penalizes the solutions located in densely populated solution regions, and a clustering strategy, which control the size of elitist list of good initial solutions.

\section{Conclusions}

Today, for most manufacturing companies, it is very important to reduce energy consumption (or use the energy resources efficiently) in their systems due to not only environmental reasons but future competitiveness of the companies. Although this has become a critical issue, very little research related to energy savings or energy reduction has been done on PCB manufacturing. To be more competitive in the global marketplace, PCB manufacturers should respond to emerging trends such as energy efficiency as well as improve the ability to produce the high-quality products.

In this research, we aimed to finding an optimal operating condition which minimize energy consumption, and maximize the non-defective probability in the PCB lamination process simultaneously. This type of problem frequently rises in PCB manufacturing, and it might be considerably difficult to obtain an optimal solution within reasonable computation time. Thus, we considered three key parameters which affect energy consumption and quality of the PCB panel manufactured in the lamination process, and applied an effective experimental design technique (3-level cuboidal design) to collect data. Based on the experimental results, we constructed regression models for two measures, energy consumption and non-defective probability of PCB panel. We then developed and applied two simple heuristic approaches and the desirability function optimization method to this problem in order to obtain solutions.

These kinds of algorithmic methods can be applied across a wide range of different processes or industries as well as manufacturing processes, for example, heat conservation in the steel manufacturing industry. However, it is still necessary to build up further knowledge or gather more detailed information on the manufacturing processes in order to derive optimal operating conditions. Furthermore, in order to improve the computational efficiency, we should explore more efficient search methods or modify current developed algorithms.

\section{ACKNOWLEDGEMENT}

This research was supported by the Chung-Ang University research grant in 2013, and by Basic Science Research Program through the National Research Foundation of Korea (NRF) funded by the Ministry of Science, ICT and Future Planning (2012RIAIAI011802).

\section{REFERENCES}

1. Laosiritaworn, W. S. and Aoonchan, P. "Multi-panel Lamination Process Optimization with Design of Experiment," Proc. of the World Congress on Engineering and Computer Science, Vol. 2, pp. 1383-1387, 2012.

2. Groover, M. P., "Fundamentals of Modern Manufacturing: Material, Processes, and Systems,” Prentice Hall, pp. 832-847, 1996.

3. Edwards, P. R., "Manufacturing Technology in the Electronics Industry," London Chapman \& Hall, pp. 5-6, 1991.

4. Park, Y-J. and Lee, G-B., "Application of Heuristic Approaches to Minimization of Energy Consumption in Inner Layer Scrubbing Process in PCB Manufacturing,” Int. J. Precis. Eng. Manuf., Vol. 13, No. 7, pp. 1059-1066, 2012.

5. Park, C. -W., Kwon, K. -S., Kim, W. -B., Min, B. -K., Park, S. -J., et al., "Energy Consumption Reduction Technology in Manufacturing- 
A Selective Review of Policies, Standards, and Research,” Int. J. Precis. Eng. Manuf., Vol. 10, No. 5, pp. 151-173, 2009.

6. Lee, J. Y., Kang, H. S., and Noh, S. D., "Simulation-based Analysis for Sustainability of Manufacturing System," Int. J. Precis. Eng. Manuf., Vol. 13, No. 7, pp. 1221-1230, 2012.

7. Goosey, M. and Kellner, R., "Energy Conservation and Related Best Practices in Printed Circuit Board(PCB) Manufacturing," Circuit World, Vol. 36, No. 1, pp. 38-42, 2010.

8. Liow, J. L., "Mechanical Micromachining: A Sustainable Microdevice Manufacturing Approach?" Journal of Cleaner Prodoction, Vol. 17, No. 7, pp. 662-667, 2009.

9. Luo, X., Cheng, K., Holt, R., and Liu, X., "Modeling Flank Wear of Carbide Tool Insert in Metal Cutting," Wear, Vol. 259, No. 7, pp. 1235-1240, 2005.

10. Rajemi, M., Mativenga, P., and Aramcharoen, A., "Sustainable Machining: Selection of Optimum Turning Conditions based on Minimum Energy Considerations," Journal of Cleaner Production, Vol. 18, No. 10, pp. 1059-1065, 2010.

11. Pusavec, F., Krajnik, P., and Kopac, J., “Transitioning to Sustainable Production-Part I: Application on Machining Technologies,” Journal of Cleaner Production, Vol. 18, No. 2, pp. 174-184, 2010.

12. Lee, G-B., Ko, M-J., and Ku, T-J., "Analysis of Energy Efficiency in PCB Manufacturing Process,” Int. J. Precis. Eng. Manuf., Vol. 13, No. 7, pp. 1215-1220, 2012.

13. Park, H-S. and Dang, X-P., "Optimization of the In-line Induction Heating Process for Hot Forging in Terms of Saving Operating Energy,” Int. J. Precis. Eng. Manuf., Vol. 13, No. 7, pp. 1085-1093, 2012.

14. Or, I. and Duman, E., "Optimization Issues in Automated Production of Printed Circuit Boards: Operations Sequencing, Feeder Configuration and Load Balancing Problems," Proc. of the IEEE Conference on Emerging Technologies and Factory Automation, pp. 227-232, 1996.

15. Kazaz, B. and Altýnkemer, K., "Optimization of Multi-feeder (depot) Printed Circuit Board Manufacturing with Error Guarantees," European Journal of Operational Research, Vol. 150, No. 2, pp. 370-394, 2003.

16. Doniavi, A., Mileham, A. R., and Newnes, L. B., "A Systems Approach to Photolithography Process Optimization in an Electronics Manufacturing Environment," International Journal of Production Research, Vol. 38, No. 11, pp. 2515-2528, 2000.

17. Ancău, M., "The Optimization of Printed Circuit Board Manufacturing by Improving the Drilling Process Productivity," Computers \& Industrial Engineering, Vol. 55, No. 2, pp. 279-294, 2008.

18. Ancãu, M., "The Processing Time Optimization of Printed Circuit Board," Circuit World, Vol. 35, No. 3, pp. 21-28, 2009.

19. Eldos, T., Kanan, A., and Aljumah, A., "Solving the Printed Circuit
Board Drilling Problem by Ant Colony Optimization Algorithm," Proc. of the World Congress on Engineering and Computer Science, Vol. I, pp. 23-25, 2013.

20. Kanchanasuttisang, P. and Luangpaiboon, P., "Sequential Multiple Response Optimization for Manufacturing Flexible Printed Circuit," American Journal of Applied Sciences, Vol. 9, No. 5, pp. 772-778, 2012.

21. Kumar, R. and Li, H., "Assembly Time Optimization for Pcb Assembly," Proc. of the IEEE on American Control Conference, Vol. 1, pp. 306-310, 1994.

22. Landers, T. L., "Electronic Manufacturing Process," Prentice-Hall, pp. 307-308, 1994.

23. Altinkemer, K., Kazaz, B., Köksalan, M., and Moskowitz, H., "Optimization of Printed Circuit Board Manufacturing: Integrated Modeling and Algorithms," European Journal of Operational Research, Vol. 124, No. 2, pp. 409-421, 2000.

24. Park, T. and Kim, N., "A Dynamic Programming Approach to PCB Assembly Optimization for Surface Mounters," International Journal of Control Automation and Systems, Vol. 5, No. 2, Paper No. 192, 2007.

25. Ho, W., Ji, P., and Dey, P. K., "Optimization of PCB Component Placements for the Collect-and-place Machines," International Journal of Advanced Manufacturing Technology, Vol. 37, No. 7-8, pp. 828-836, 2008.

26. Haimes, Y. Y., Lasdon, L. S., and Wismer, D. A., "On a Bicriterion Formulation of the Problems of Integrated System Identification and System Optimization,” Proc. of the IEEE Transactions on System, Man, and Cybernetics, Vol. 1, No. 3, pp. 296-297, 1971.

27. Mavrotas, G., "Effective Implementation of the $\varepsilon$-constraint Method in Multi-objective Mathematical Programming Problems," Applied Mathematics and Computation, Vol. 213, No.2, pp. 455-465, 2009.

28. Koski, J. and Silvennoinen, R., "Norm Methods and Partial Weighting in Multicriterion Optimization of Structures," International Journal for Numerical Methods in Engineering, Vol. 24, No. 6, pp. 1101-1121, 1987.

29. Kim, I. Y. and De Weck, O., "Adaptive Weighted Sum Method for Multiobjective Optimization: A New Method for Pareto Front Generation," Structural and Multidisciplinary Optimization, Vol. 31, No. 2, pp. 105-116, 2006.

30. Deb, K., "Nonlinear Goal Programming using Multi-objective Genetic Algorithms," Journal of the Operational Research Society, Vol. 52, No. 3, pp. 291-302, 2001.

31. Lin, D. K. and Tu, W., "Dual Response Surface Optimization," Journal of Quality Technology, Vol. 27, No. 1, pp. 34-39, 1995.

32. Vining, G. G. and Myers, R. H., "Combining Taguchi and Response Surface Philosophies: A Dual Response Approach," Journal of Quality Technology, Vol. 22, No. 1, pp. 38-45, 1990.

33. Derringer, G., "Simultaneous Optimization of Several Response Variables," Journal of Quality Technology, Vol. 12, pp. 214-219, 1980. 
34. Coello Coello, C. C., Van Veldhuize, D. A., and Lamont, G. B., "Evolutionary Algorithms for Solving Multi-objective Problems," Kluwer Academic Publishers, 2002.

35. John, H., “Adaptation in Natural and Artificial Systems," MA: MIT Press, pp. 89-118, 1992.

36. Goldberg, D. E., "Genetic Algorithms in Search, Optimization, and Machine Learning,” Addison-Wesley Reading Menlo Park, 1989.

37. Whitley, D., "A Genetic Algorithm Tutorial," Statistics and Computing, Vol. 4, No. 2, pp. 65-85, 1994.

38. Montgomery, D. C., Peck, E. A., and Vining, G. G., "Introduction to Linear Regression Analysis,” John Wiley \& Sons, $5^{\text {th }}$ Ed., pp. 67-73, 2012.

39. Montgomery, D. C., "Design and Analysis of Experiments," Wiley, $8^{\text {th }}$ Ed., pp. 174-177, 2013. 